\title{
Intrasacral meningocele in the pediatric population
}

\author{
Clinical article
}

\author{
Subash Lohani, M.D., ${ }^{1}$ Diana P. Rodriguez, M.D., ${ }^{2}$ Hart G. W. Lidov, M.D., ${ }^{3}$ \\ R. Michael Scott, M.D., ${ }^{1}$ and Mark R. Proctor, M.D. ${ }^{1}$ \\ Departments of ${ }^{1}$ Neurosurgery, ${ }^{2}$ Neuroradiology, and ${ }^{3}$ Neuropathology, Boston Children's Hospital, Boston, \\ Massachusetts
}

Object. Intrasacral meningoceles are rare cystic lesions that can cause focal compression within the bony sacral canal. Their mechanisms are poorly understood, but most intrasacral meningoceles appear to be intrasacral extradural cysts caused by arachnoid herniating through a small dural defect in the caudal end of the thecal sac. As opposed to perineural cysts, they are not associated with an exiting nerve root. When symptomatic, they can cause sacral pain or sacral nerve root dysfunction due to local compression.

Methods. This is a retrospective series from Boston Children's Hospital. All patients in whom symptomatic intrasacral meningocele that required surgical treatment was diagnosed between May 1994 and March 2011 were included in the study. Spine MRI was the diagnostic modality of choice. All patients underwent sacral exploration, with ligation and obliteration of the cyst. Resected cyst wall was subjected to pathological examination.

Results. There were 13 patients (11 boys and 2 girls) who underwent operation for intrasacral meningocele. The median age was 8 years (range 5 months-16 years). The most common presenting symptom was back pain (in 5) often described as deep tail bone pain, followed by urinary incontinence (3) and constipation (2). Three patients had evidence of associated tethered cord on MRI studies. Four patients were asymptomatic and their diagnosis was made following imaging for other reasons; they were surgically treated because of the increasing size of the lesion or association with other congenital lesions. Most patients had symptomatic improvement after surgery.

Conclusions. Intrasacral meningoceles are rare lesions that may result from a congenital dural weakness and a resultant arachnoid diverticulum. They present in childhood either incidentally or with symptoms secondary to nerve root compression. Identification of the point of herniation through the dura mater and ligation of the lesion provides cyst cure and resolution of symptoms in most patients.

(http://thejns.org/doi/abs/10.3171/2013.3.PEDS12519)

KeY Words • intrasacral meningocele • sacral canal • congenital cyst

$\mathrm{T}$ ThIs study was undertaken to categorize the clinical presentation, radiological appearance, surgical anatomy, and operative results in pediatric patients undergoing operation for intrasacral meningocele. This series focuses only on the occult intrasacral meningoceles, defined as extradural cysts associated with herniating arachnoid, but not associated with an exiting nerve root. Due to their relative rarity, the literature on intrasacral meningoceles has been limited to case reports, and we believe that our report represents the first published operative series of intrasacral meningoceles. Perineural cysts, defined as cysts associated with an exiting nerve

Abbreviations used in this paper: GFAP $=$ glial fibrillary acidic protein; UDS = urodynamic studies. root, were not included, although some lesions had associated tethering pathology.

\section{Methods}

This is a retrospective study conducted at Boston Children's Hospital. After obtaining institutional review board approval, charts were reviewed to identify all patients who were surgically treated for intrasacral meningocele between May 1994 and March 2011, and to determine presenting signs and symptoms, imaging findings, anatomy at surgery, and postoperative results based on

This article contains some figures that are displayed in color online but in black-and-white in the print edition. 
imaging and clinical follow-up examinations. All surgeries were performed at our institution, predominantly by the senior author (M.R.P.).

\section{Patient Evaluation}

All patients underwent full clinical examination, and adjuvant tests such as UDS were performed based on symptoms and clinical findings. Radiographic studies included MRI of the spine for the accurate delineation of the lesion in all patients. There was a single instance each in which spine ultrasound and CT myelography was also performed. The UDS were done if the patient had urological symptoms.

\section{Surgical Procedure}

All operations were performed with the patients under general anesthesia. Neuromonitoring was used to monitor sacral nerve root and leg function. Identification of the spinal level and surgical opening were performed using palpable anatomical landmarks, and rarely radiography. The operating microscope was used in all cases. The goal of surgery was to identify and ligate the connection between the thecal sac and the extradural cyst. Nerve roots adherent to the exterior walls of the cyst were dissected off and a silk suture was passed around the takeoff of the cyst at the base of the thecal sac. The cyst was then opened and explored internally to confirm that it did not contain any neural elements. The circumference of the cyst was electrically stimulated to be certain there were no active motor roots, and after the lesion was definitively isolated, the silk stitch was secured at the base of the cyst to eliminate its connection with the dura mater. A Valsalva maneuver was then performed by the anesthesiologist to test the competency of the ligation. In some cases the cyst walls were removed and sent for pathological examination. The newly created dead space was filled with either fat from the soft tissues or with collagen sponge (Fig. 1).

\section{Pathological Examination}

In 4 patients the cyst wall was resected and submit- ted for pathological examination. This included paraffin sections stained with $\mathrm{H} \& \mathrm{E}$, Masson Gomori trichrome, and immunostained for GFAP and epithelial membrane antigen. The sections were retrospectively reviewed by one of the authors (H.G.W.L.).

\section{Results}

There were total of 13 patients in whom operations were performed for intrasacral meningocele between May 1994 and March 2011. There were 11 boys and 2 girls (5.5:1 male/female ratio). The age range at surgery was 5 months to 16 years. The median age was 8 years.

\section{Presenting Symptoms}

The most common presenting symptom was nonradiating back pain ( 5 patients), typically described by the child as a deep tail bone pain. Three patients had urinary incontinence and their UDS demonstrated overactive bladder, intermittent flow patterns, and high postvoid residuals, all consistent with neurogenic bladder. Two patients had constipation. Lower-extremity weakness and recurrent urinary tract infection were seen in 1 patient each. Four patients were asymptomatic and diagnosed as a part of imaging for other reasons, including sacral dimple, asymmetrical gluteal cleft, and presence of other congenital anomalies like multiple segmentation and fusion vertebral anomalies. Additional findings that we observed on clinical examination were sacral dimple in 3 patients ( 2 with benign sacral dimple and 1 associated with asymmetrical gluteal cleft) and a dermal sinus tract in 1 child. One patient in our series developed an intrasacral meningocele after a traumatic epidural spinal anesthetic and presented with subtle lower-extremity motor weakness and depressed ankle jerk, which partially improved after surgery. The clinical findings in these patients are summarized in Table 1.

\section{Surgical Findings}

In all patients a well-circumscribed cyst with smooth
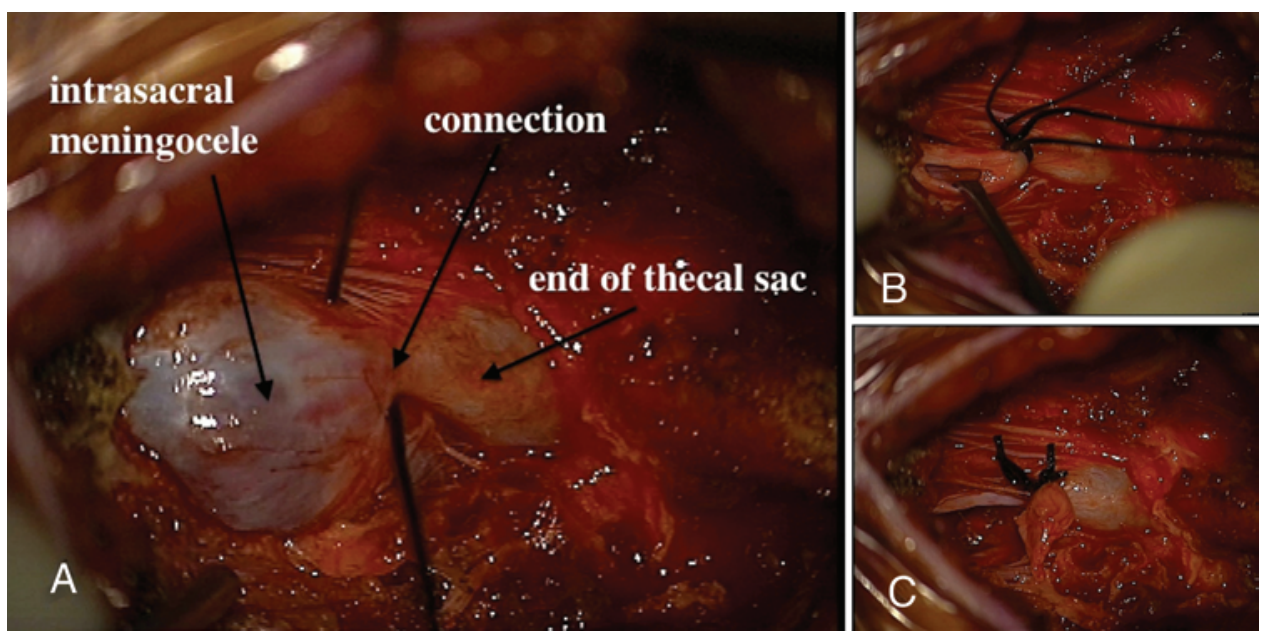

FIG. 1. A: Intraoperative photograph showing a cyst (left side of panel) at the distal end of the thecal sac; a silk ligature is passed around its connection with the dura mater. B: The silk tie is secured and the cyst is opened and explored. C: The cyst wall is resected after confirming that it is free of nerve roots. 
Intrasacral meningocele in the pediatric population

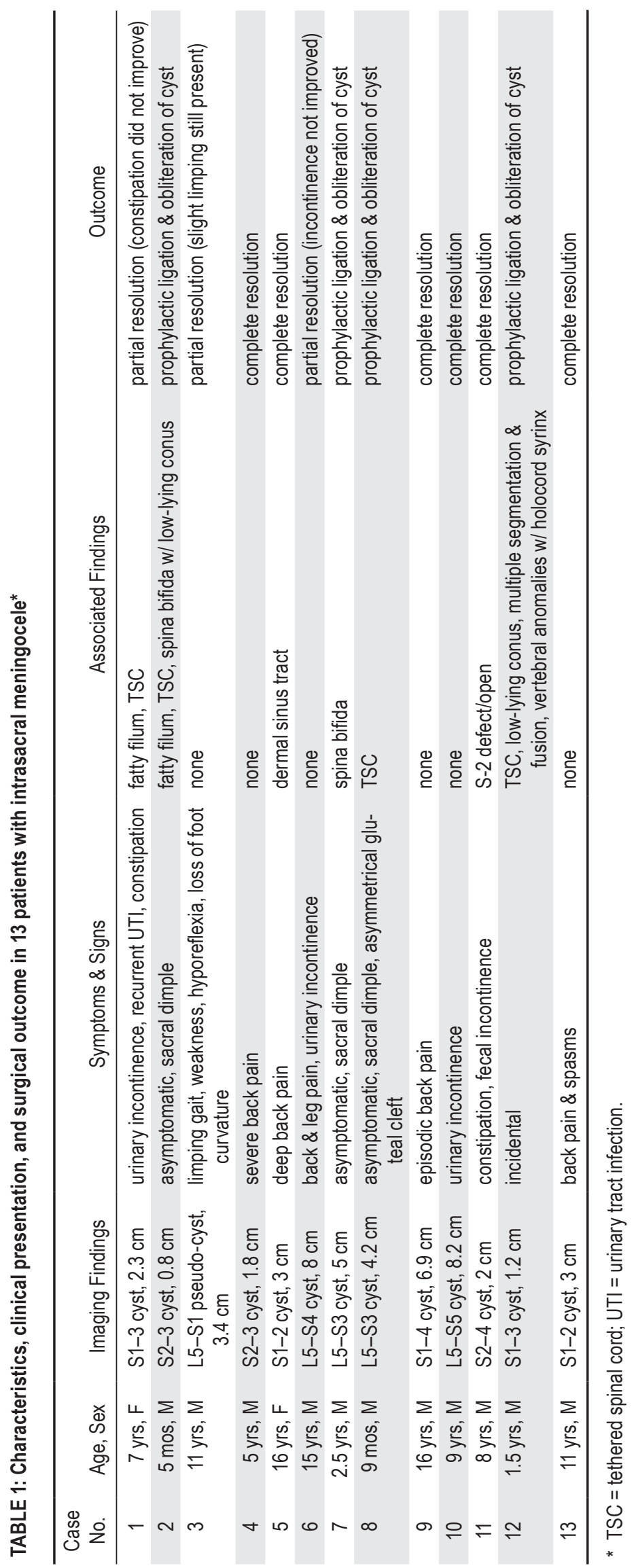


contours centered within the sacral spinal canal was identified. The craniocaudal length of these cysts ranged from $0.8 \mathrm{~cm}$ to $8.2 \mathrm{~cm}$. Five lesions extended cephalad to the level of the lumbosacral junction. Neural elements were not seen within any of these cysts. The adjacent nerve roots were often displaced by the cyst, and the largest lesions caused mass effect on the distal thecal sac in addition to the exiting nerve roots.

\section{Imaging Modalities}

Initial workup consisted of MRI studies of the spine. The MRI spine studies consisted of the following sequences: conventional T1- and T2-weighted spin-echo MR images obtained in sagittal and axial planes. Occasionally 3D FIESTA and diffusion-weighted imaging of the spine was performed. The 3D FIESTA images suggested the site of possible connection of the cyst to the distal thecal sac in 5 patients. In 2 patients, the lesions were evaluated with diffusion-weighted imaging and did not demonstrate decreased diffusivity. The fluid within these cysts followed CSF signal intensity on all sequences. Three patients had documented progressive enlargement on serial follow-up imaging before surgery.

In 6 patients, remodeling of the spinal canal resulted in an expansion of the canal, with scalloping of the dorsal aspect of the vertebral bodies. There was no evidence of neuroforaminal enlargement, and none of these lesions extended beyond the margins of the sacrum.

Four patients presented with one of the following associated intraspinal abnormalities: thickened filum; a distal sacral intraspinal lipoma with a tethered cord terminating at the L-4 level; multiple segmentation and fusion vertebral anomalies with syrinx and tethered cord at the L-4 level (Fig. 2); and a dermal sinus tract. A bony spina bifida occulta was present in 3 patients.

\section{Postoperative Complications}

One patient had minor cellulitis of the wound edges on postsurgical Day 10 that required local wound care.

\section{Patient Outcomes}

Most of the patients had partial to complete symptomatic improvement after the surgery. Pain improved in all 5 patients, and urinary symptoms improved in 2 of 3 patients, confirmed by follow-up UDS. As an example, UDS in 1 patient showed multiple overactive bladder contractions and a significant postvoid residual. After surgery there was marked improvement in the overactive bladder, with only a few overactive contractions, and the patient achieved normal voiding dynamics with near-complete bladder emptying. One patient whose urinary symptoms did not improve following surgery was found to have an anatomical urethral obstruction that required a subsequent urological procedure. Constipation improved in 1 of 2 patients. Preoperative leg weakness in 1 patient with an iatrogenic cyst improved after cyst excision, although the ankle jerk continued to remain depressed. Postoperative MRI studies were done in 10 of 13 patients (Fig. 3), demonstrating cyst obliteration in all cases.

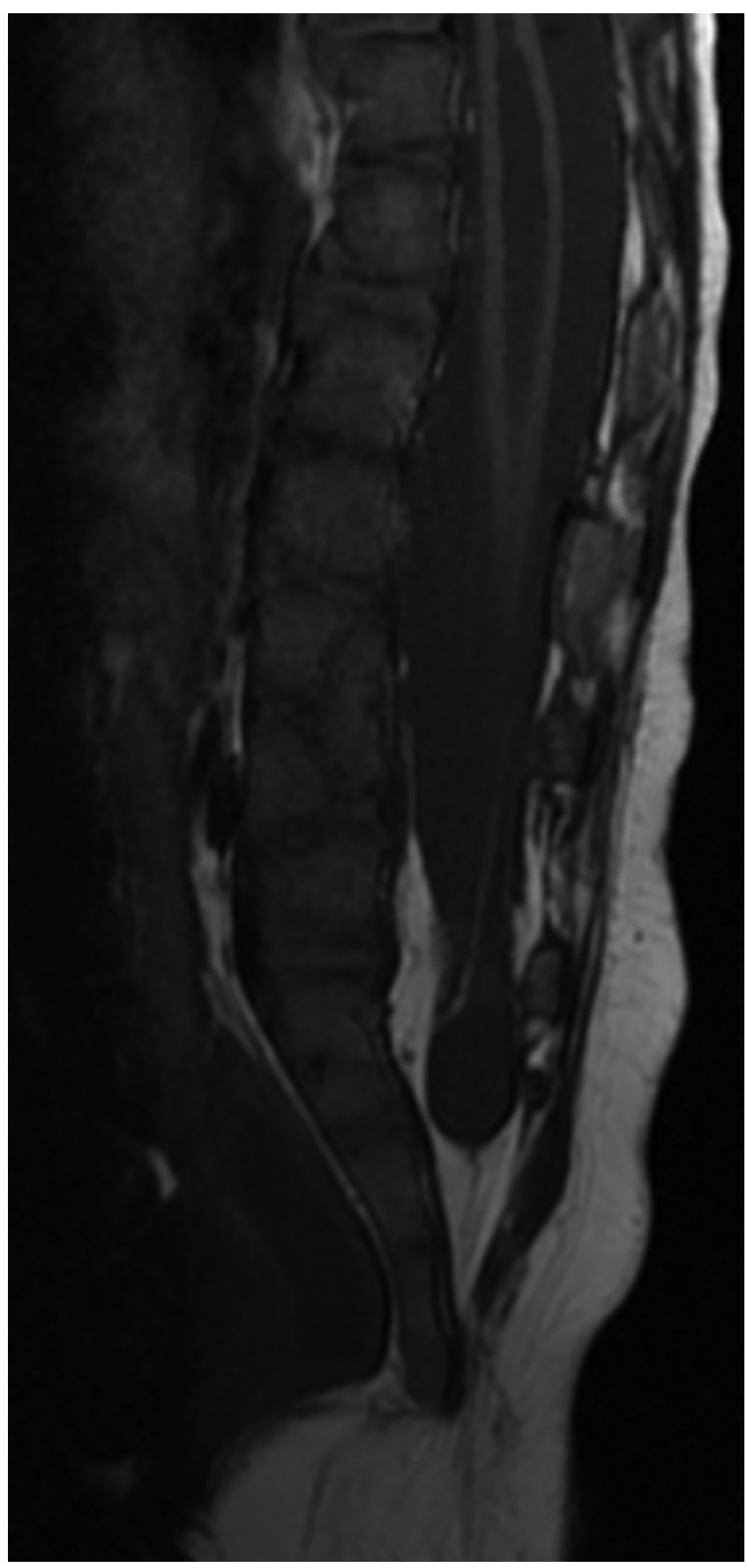

FIG. 2. An MR image of the lumbosacral spine obtained in a 1.5-yearold boy demonstrating an intrasacral meningocele associated with tethered cord from a fatty filum terminale and congenital bony anomalies.

\section{Pathological Findings}

In 4 cases, the walls of the cyst were sent for pathological examination. Specimens had a characteristic microscopic appearance. In the paraffin-embedded histological sections there were ribbons of variably flattened membranes, and it was difficult to distinguish between the internal luminal surface and external "epidural" surface: a lacy and poorly defined layer with cuboidal epithelium was interpreted as the internal layer, whereas a fibrovascular connective tissue with an outer thickened 


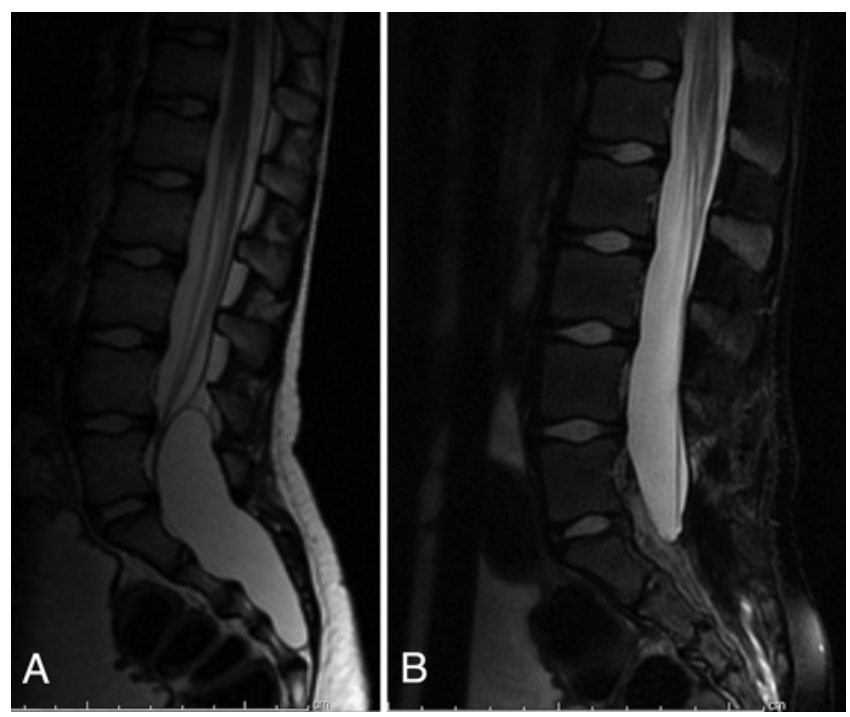

FIG. 3. Preoperative (A) and postoperative (B) MRI studies obtained in a 9 -year-old boy presenting with urinary incontinence. He experienced significant improvement in urinary function.

layer of densely packed collagenous membrane was interpreted as external membrane. This histological finding corresponded to what was seen at surgery, in that the cyst wall appeared to be either dura or very thickened arachnoid. Both layers, when stained with $\mathrm{H}$ \& E stain, had the appearance of collagen (Fig. 4A).

Interestingly, there were focal CNS elements found in some specimens. However, the residua of a disrupted and attenuated ependymal lining of the central canal, or other sources of glial cells, was observed in some specimens on GFAP and epithelial membrane antigen staining. Twigs of myelinated nerve fibers were found in some specimens, and these appeared to be in the outer surface of the collagenous membrane. These were thought to be associated nerve twigs, and not neuronal elements within the cyst wall.

\section{Discussion}

The term "intrasacral meningocele" has been used to denote a sac of fibrous tissue resembling dura mater that is usually lined by arachnoid, lies within an enlarged sacral spinal canal, and is attached to the caudal termination of the dural sac by a pedicle that usually permits the flow of CSF from the tip of the subarachnoid space into the meningocele. ${ }^{12}$ As discussed by Tarlov in $1938,{ }^{15}$ intrasacral meningocele was first reported by Enderle in 1932. Since then there have been numerous case reports ${ }^{3,6,8,11-13,15,16}$ but no pediatric surgical case series. Intrasacral meningocele is distinguished from perineural cysts by the absence of a nerve root within the cyst. Various terminologies in the literature include occult intrasacral meningocele, intrasacral cyst, intrasacral extradural arachnoid cyst, intraspinal meningocele, or giant sacral meningeal diverticula. These cysts are occasionally discovered incidentally, but may also present with back pain or bowel and bladder difficulties from local compression of the nerve roots by the cyst.

In 1988 Nabors et al. ${ }^{10}$ classified spinal meningocele cysts into 3 categories: Type I, extradural cysts without spinal nerve root fibers; Type II, extradural cysts with spinal nerve root fibers; and Type III, intradural cysts. By this system, intrasacral meningoceles would be classified as Type I spinal meningocele. This classification has not been widely accepted, and indeed intraspinal cysts remain a relatively rare condition. This series focuses only on the Type I cyst-the extradural occult intrasacral meningocele not associated with an exiting nerve root.

Although often confused with perineural (Tarlov) cysts, intrasacral meningoceles differ in that they do not contain neural elements and are found more centrally in the spinal canal, whereas Tarlov cysts typically develop more laterally along a nerve root. The intrasacral meningocele may therefore be thought of as a sacral extradural arachnoid cyst. ${ }^{9}$ In these lesions the arachnoid herniates through a small, probably congenital, dural defect in the caudal end of the thecal sac. This arachnoid diverticulum
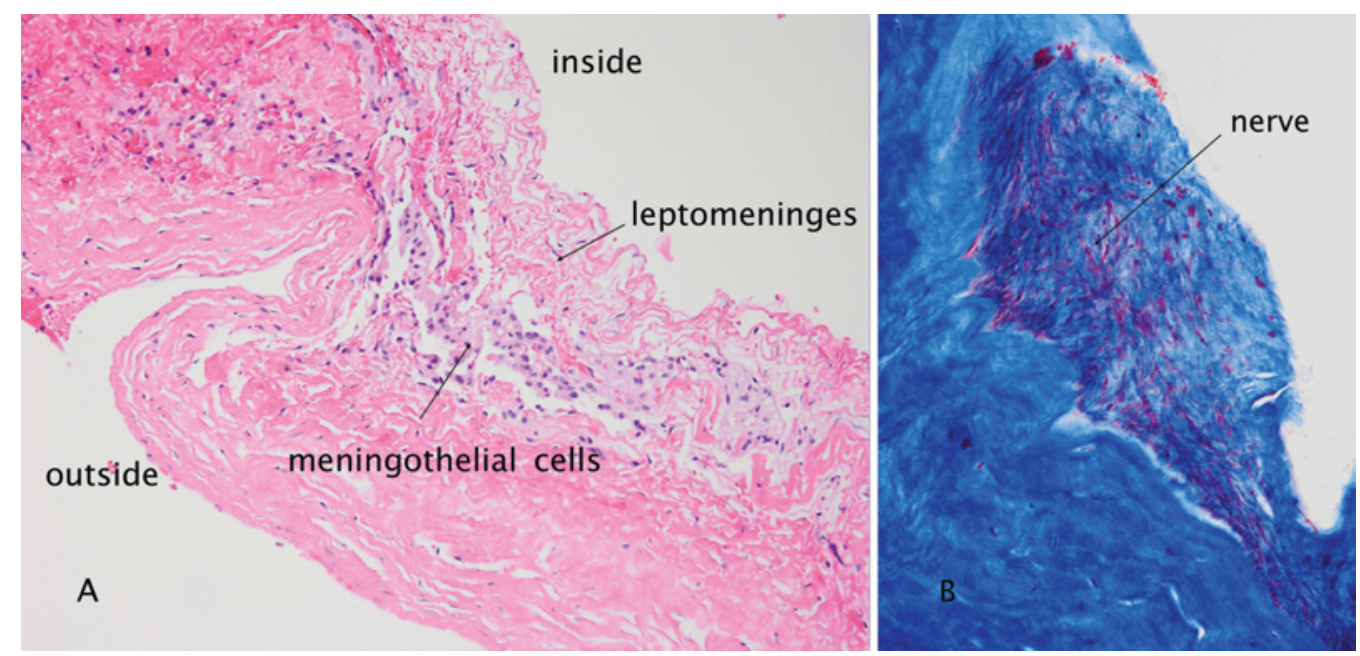

Fig. 4. Photomicrographs. A: Section of cyst wall demonstrating the outer thickened collagenous layer consistent with dura or thickened arachnoid (upper left) and an inner lacy membrane of leptomeninges (lower right). B: Section of the cyst wall demonstrating a nerve twig with scattered myelinated fibers (red). $H \& E(A)$ and trichrome $(B)$ stains, original magnification $\times 40$. 
progressively increases in size because of egress of CSF into the cyst with erect posture, which may be related to the ball-valve effect.

Valsalva maneuvers that occur during daily activities may also increase the volume of the cyst..$^{15}$ If the cyst is small enough that the takeoff of the lesion is apparent on MRI this central location seems to be an important differentiating feature, and often an intrasacral meningocele can be distinguished from a nerve root cyst on this basis (Fig. 5).

Various theories of pathogenesis have been proposed in the formation of these lesions; they include the following. 1) Dysraphic theory: failure of neural tube closure or failed closure of the surrounding mesoderm because of developmental arrest during various stages of embryogenesis. 2) Acquired dural disruption theory: cyst is the result of a dural defect caused by direct traumatic injury or operative intervention, with resultant herniation of the arachnoid through the defect. 3) Proliferative theory: open neural tube in intrasacral meningocele may be the result of local overgrowth that interferes with closure, rather than a consequence of developmental arrest. 4) Failure of meningeal sac ascension: meningeal sac fails to ascend from its embryonic coccygeal position to its normal adult position at S-2. ${ }^{8}$ Of these, the most accepted theory today is that the condition is due to a primary mesenchymal defect involving the dura. An arachnoid hernia forms through the dural defect, and with time it increases in size and produces bony changes secondary to pressure erosion. ${ }^{13}$

Although 3 of the 4 theories propose a congenital origin, it is likely that the cyst progresses over time due to hydrostatic pressure, and the diagnosis in most reported cases is established during adulthood. ${ }^{8}$ Certainly this would be supported by the fact that 3 of the cysts in

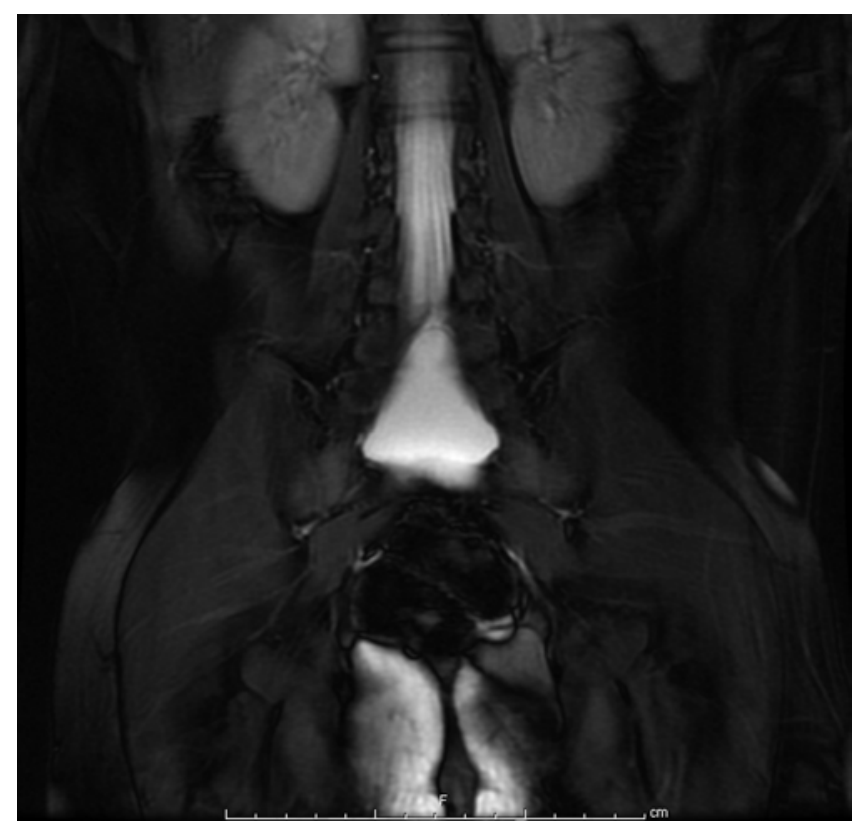

FIG. 5. Coronal section of an MR image of the lumbosacral spine showing the rostral end of an intrasacral meningocele at the distal end of the thecal sac; the cyst is primarily midline in origin. our series were observed to expand over time. As with many other conditions, the greater availability of MRI has resulted in higher diagnostic rates in the pediatric population, and increasingly, asymptomatic cases are being diagnosed incidentally during MRI. There were 4 incidentally discovered cysts in our series. Intrasacral meningocele has been diagnosed in patients as young as 5 months because the child underwent imaging for other reasons such as sacral dimple.

Consistent with the possible congenital origin of these lesions, intrasacral meningocele may occur in association with other anomalies like sacral vertebral anomalies, ${ }^{11}$ diastematomyelia, ${ }^{8}$ or tethered spinal cord..$^{3,6,8,12}$ There have been previous case reports of associated thickened filum ${ }^{3,6}$ and lumbar disc prolapse. ${ }^{1,16}$ In our series, 3 had associated tethering, 3 had spina bifida occulta, 1 had vertebral segmentation anomaly, and 1 had a dermal sinus tract.

In the literature, the most common presenting symptoms are pain and altered bowel and bladder habits. Typical complaints are chronic, intermittent low-back pain. Nerve root compression by the large cyst is probably the cause of the patient's symptoms, although often the pain does not radiate beyond a deep "tail bone" pain. ${ }^{16}$ Longstanding episodic gluteal pain and progressive muscle wasting has also been described..$^{9}$ Our experience concurs with the literature, with back pain being the most common complaint (5 patients). Aggravation of symptoms by activity or Valsalva maneuver is reported. ${ }^{4}$ Altered bladder or bowel habits, including urinary incontinence and constipation, are probably related to compression of the sacral roots by the full and firm meningocele. ${ }^{11}$ This was the second most common presentation in our series. In other series of spinal arachnoid cysts, pain is consistently the most common symptom, although bowel and bladder disturbance is clearly more related to those cysts in the sacral region. ${ }^{2}$

Rarely, intrasacral meningoceles can present after spine trauma with subsequent CSF leakage and symptoms related to intracranial hypotension, ${ }^{7}$ presumably related to rupture of a preexisting cyst. At times, they may also present with pathological fracture of the sacrum due to erosion of the bone by the cyst as it enlarges. A minor trauma may precede such presentation and is more likely in adults than in children. ${ }^{5}$

\section{Imaging Modalities}

Magnetic resonance imaging of the spine is the imaging modality of choice to establish the diagnosis of intrasacral cyst. In addition to providing fine anatomical detail for preoperative planning, MRI can differentiate intrasacral meningocele from other lesions. The typical imaging findings of intrasacral meningocele consist of an extradural cyst with smooth contours located within the sacral spinal canal (adjacent to the distal thecal sac) that follows CSF signal intensity on all sequences. No neural elements are seen within the cyst. However, the site of connection between the cyst and the thecal sac (isthmus or pedicle) can be difficult to determine using MRI. In our experience, if the apex of the lesion appears midline, it is more likely to be an intrasacral meningocele rather than a perineural cyst. In contrast, a perineural cyst is usually located ec- 


\section{Intrasacral meningocele in the pediatric population}

centrically within a neural foramen and has an associated enlargement of the foramen as well as dilation of the nerve root meningeal sleeve. Although conventional MRI findings are usually sufficient for diagnosis, 3D-FIESTA images generate higher-resolution images, and thus enhanced anatomical detail that can occasionally depict the connection between the cyst and the dura. Because intrasacral meningoceles do not enhance, administration of contrast material is not indicated. Diffusion-weighted imaging may be helpful in differentiating an intrasacral meningocele from an epidermoid tumor.

Myelography and CT myelography have been used to diagnose intrasacral cysts and can occasionally depict the connection point between the cyst and the thecal sac, depending on the size of the opening. These methods, however, are invasive, time-consuming, painful, and are associated with ionizing radiation; a significant concern when performing imaging in the pediatric population. Because of these several disadvantages and the lack of significant added information, myelography and CT myelography are rarely used in the pediatric population.

Plain radiographs can sometimes reveal remodeling of the sacral canal, or other bony abnormalities such as segmentation anomaly, fusion vertebral anomalies, and spinal dysraphism.

Ultrasound plays a role in the identification of intrasacral meningoceles in infants if it is performed before 3-4 months of age. In our cohort, 1 patient underwent an ultrasonography study of the spine to exclude associated intraspinal abnormality in the clinical setting of a sacral dimple and asymmetrical gluteal folds. This method effectively demonstrated the cyst.

Based on imaging findings, other differential diagnoses may include dorsal meningocele and dural ectasia. A dorsal meningocele (that is, a "true" meningocele) will show protrusion of the meningeal sac through a dorsal dysraphism with extension into the paraspinal soft tissues. Dural ectasia usually involves both the lumbar and sacral spine, with or without the presence of lateral components, and is not separated from the thecal sac by a pedicle. As- sociated abnormalities could suggest this diagnosis, depending on the etiology (for example, neurofibromatosis Type 1, Marfan disease).

There has been 1 report of intrasacral meningocele that contains documented serial MRI confirmation of enlargement of the cyst. ${ }^{8}$ Three patients in our series had progressive increase in cyst size on serial imaging, for which the decision was made in favor of surgical intervention.

\section{Treatment Procedure}

When treatment is required for growing or symptomatic lesions, surgical ligation and obliteration of the cyst is the only known procedure to treat this disorder. We have found that careful microsurgical exploration with identification and ligation of the fistula is the best way to treat these lesions. Generally the exposure is limited to the rostral end of the meningocele. Internally, one will often see a small aperture in the dura with arachnoid sneaking through, and it can create a 1-way ball-valve effect. Our approach is similar to techniques that have been reported previously. ${ }^{11,13}$ Postoperative MRI studies will reliably assess the success of surgery.

Intrasacral meningocele does not necessarily require surgical treatment. ${ }^{1}$ Surgery is indicated when there are progressive symptoms or when an increase in the size of the cyst is demonstrated on radiological follow-up. ${ }^{16} \mathrm{We}$ generally would not recommend surgery for an asymptomatic cyst unless enlargement has been documented or the child requires surgery for a related spinal disorder such as cord tethering. It is hard to quantify what percentage of cysts will enlarge. We have seen 3 lesions enlarge radiographically over relatively short spans of 1-3 years, but a small number of others have remained stable (Fig. 6). Overall, the rarity of the condition will make it hard to establish a firm guideline on how likely it is to progress in those not presenting with symptoms. In our series 9 patients were symptomatic. Operations in 3 patients were performed prophylactically, 2 of which were in conjunction with detethering. Three patients, 1 of whom was as-
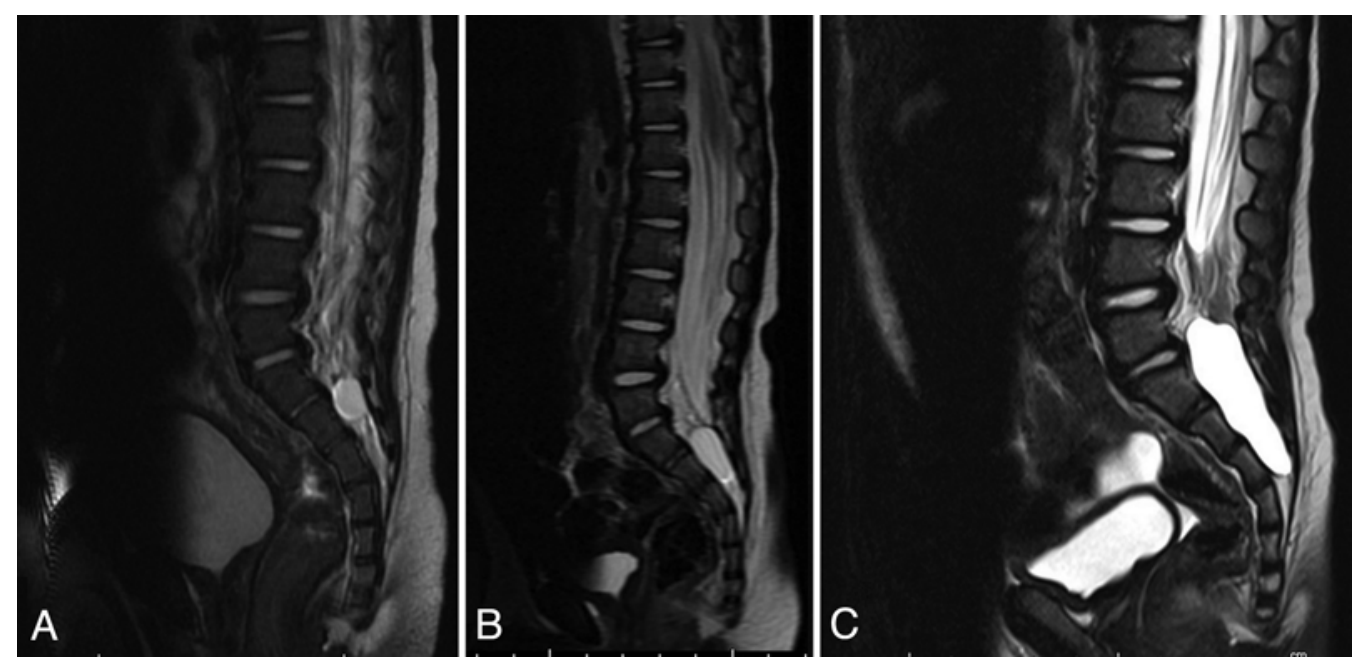

FIG. 6. Series of MRI studies of the lumbosacral spine obtained at 4 months (A), 7 months (B), and 21 months (C), demonstrating rapid internal enlargement of an intrasacral meningocele in a boy in whom the imaging was originally performed for a sacral dimple. 
ymptomatic, underwent surgery progressive enlargement of the cyst.

\section{Pathological Findings}

In general the cyst specimen is an entirely collagenous, non-CNS membrane. It remains unclear if the dense layer of collagen seen in some of the specimens is attenuated dura or thickened reactive arachnoid. Nerve roots were not an integral part of the cyst walls. These findings are consistent with the few descriptions almost exclusively based on $\mathrm{H} \& \mathrm{E}$ staining in the literature..$^{10,14}$

Interestingly, what was present in these specimens was a simple cuboidal epithelium that is GFAP positive, consistent with the dilated remains of the ependymal lining of the central canal. This would be consistent with a congenital origin or herniation of CNS elements into the defect. These cellular components, although rather different in overall architecture, are similar to the constituents of a simple tethered cord. One might speculate that some of these specimens arise as a cystic dilation of the filum terminale, and in some of our cases the filum could in fact be followed into the the meningocele.

\section{Conclusions}

Intrasacral meningoceles are sacral extradural arachnoid diverticulum that are probably of congenital origin and may either be detected incidentally or present with symptoms of deep tail bone or leg pain, motor dysfunction, or bowel and bladder difficulties. Males seem to be preferentially affected. An MRI study of the lumbosacral spine is the investigation of choice. Surgical repair with ligation and obliteration of the cyst is the preferred treatment for symptomatic or growing lesions, and symptomatic improvement is generally noted after the surgery.

\section{Disclosure}

The authors report no conflict of interest concerning the materials or methods used in this study or the findings specified in this paper.

Author contributions to the study and manuscript preparation include the following. Conception and design: Proctor. Acquisition of data: Lohani, Rodriguez, Lidov. Analysis and interpretation of data: Lohani. Drafting the article: Lohani, Rodriguez, Lidov. Critically revising the article: all authors. Reviewed submitted version of manuscript: all authors. Approved the final version of the manuscript on behalf of all authors: Lohani. Administrative/techni$\mathrm{cal} /$ material support: Proctor. Study supervision: Proctor.

\section{References}

1. Bayar MA, Yidiz B, Buharali Z: Management problems in cases with a combination of asymptomatic occult intrasacral meningocele and disc prolapse. Acta Neurochir (Wien) 108:67-69, 1991
2. Bond AE, Zada G, Bowen I, McComb JG, Krieger MD: Spinal arachnoid cysts in the pediatric population: report of 31 cases and a review of the literature. Clinical article. J Neurosurg Pediatr 9:432-441, 2012

3. Doi H, Toyoda I, Matsumoto K, Imai S, Sekimizu M: Occult intrasacral meningocele with tethered cord-case report. Neurol Med Chir (Tokyo) 35:321-324, 1995

4. Doty JR, Thomson J, Simonds G, Rengachary SS, Gunby EN: Occult intrasacral meningocele: clinical and radiographic diagnosis. Neurosurgery 24:616-625, 1989

5. Fardon DF: Intrasacral meningocele complicated by transverse fracture. A case report. J Bone Joint Surg Am 62:839841,1980

6. Feigenbaum F: Giant sacral meningeal diverticulum containing a thickened filum with lipoma in an adult with spinal cord tethering. Case report and review of the literature. J Neurosurg Spine 9:281-284, 2008

7. Kihara T, Mitsueda T, Ito K, Miyata M: Intracranial hypotension caused by traumatic intrasacral meningocele. J Neurol Neurosurg Psychiatry 75:658, 2004

8. Kiliçkesmez O, Barut Y, Tasdemiroglu E: Expanding occult intrasacral meningocele associated with diastematomyelia and multiple vertebral anomalies. Case report. J Neurosurg 101 (1 Suppl):108-111, 2004

9. Mishra GP, Sharma RR, Lad SD, Pawar SJ, Mahapatra AK: Gluteal neuralgia-unusual presentation in an adult with intrasacral meningocele: a case report and review of literature. Neurol India 48:279-281, 2000

10. Nabors MW, Pait TG, Byrd EB, Karim NO, Davis DO, Kobrine AI, et al: Updated assessment and current classification of spinal meningeal cysts. J Neurosurg 68:366-377, 1988

11. Nishio Y, Hamada H, Kurimoto M, Hayashi N, Hirashima Y, Endo S: A case of occult intrasacral meningocele presented with atypical bowel symptoms. Childs Nerv Syst 20:65-67, 2004

12. Parmar H, Shah J, Varma R, Patkar D: Intrasacral meningocele with tethered cord syndrome. J Assoc Physicians India 49:746-748, 2001

13. Rengachary SS, O'Boynick P, Karlin CA, Batnitzky S, Price $\mathrm{H}$ : Intrasacral extradural communicating arachnoid cyst: cases report. Neurosurgery 8:236-240, 1981

14. Schachenmayr W, Friede RL: The origin of subdural neomembranes. I. Fine structure of the dura-arachnoid interface in man. Am J Pathol 92:53-68, 1978

15. Tarlov IM: Perineural cysts of the spinal nerve roots. Arch Neurol Psychiatry 40:1067-1074, 1938

16. Tatagiba M, Aguiar PH, Samii M: Management of occult intrasacral meningocele associated with lumbar disc prolapse. Neurosurg Rev 17:313-315, 1994

Manuscript submitted October 19, 2012.

Accepted March 11, 2013.

Please include this information when citing this paper: published online April 19, 2013; DOI: 10.3171/2013.3.PEDS12519.

Address correspondence to: Subash Lohani, M.D., Department of Neurosurgery, Boston Children's Hospital, 300 Longwood Avenue, Hunnewell-2, Boston, Massachusetts 02115.email: subash.lohani@ childrens.harvard.edu. 